\title{
OPTICAL AND ULTRAVIOLET ABSORPTION STUDIES OF COOL GAS IN THE MILKY WAY HALO
}

\author{
L. Danly \\ Space Telescope Science Institute \\ 3700 San Martin Drive \\ Baltimore, MD 21218
}

\section{Introduction}

Of the many and various means for observing the interstellar medium, and halo gas in particular, optical and ultraviolet absorption techniques provide both unique opportunities and unique limitations. As its title indicates, this article speaks specifically to the contributions from absorption methods to our knowledge of halo gas with temperatures below $10^{5} \mathrm{~K}$. A brief description of the trade-offs and benefits of the methods is therefore useful to set the stage for interpreting the observations of halo gas. Briefly stated, the trade-offs can be described as follows:

absorption vs emission studies. One obvious benefit from absorption studies is that they provide limits on the distance to gas. In addition, while both $\mathrm{H}^{0}$ and $\mathrm{H}^{+}$can be studied well in emission, lines from most other elements are simply too weak to be studied extensively in emission. Absorption data, on the other hand, can be obtained on many different species with a wide range of ionization state. More quantitative information is therefore available on physical properties such as abundances, depletion and ionization of gas studied in absorption. A major drawback of absorption studies is the limitation imposed by curve-of-growth effects. Quantitative determinations can be very difficult under conditions where the instrumental resolution is too poor to permit separate components to be individually resolved. Finally, absorption studies require the presence of suitable background sources against which to measure the interstellar absorption lines. Finding suitable sources can lead to limited sky-coverage, and the selection of the gas that gets studied is determined solely by the luck of the positional alignment. Furthermore, the distribution of background sources can lead to selection effects: for example, the strong stellar UV continuum required for ultraviolet absorption studies is found only in very early type stars which have a distribution strongly confined to the galactic plane.

optical vs. ultraviolet studies. Optical absorption programs have the advantage that the data is gathered from ground based telescopes which, due mostly to their larger size, can provide higher resolution spectra from shorter 
exposures. In general, more observing time is available from ground based observatories, permitting broader surveys of halo sightlines. The species available for study with optical telescopes (primarily Na I, Ca II, and Ti II) are tracers of cool gas and provide information on the depletion of gas phase atoms, but they are weak lines from elements which have low abundances relative to hydrogen and they provide no information on the presence of warm or hot gas. The currently observable ultraviolet region of the spectrum, on the other hand, contains many strong (even resonance) lines from important elements such as $\mathrm{C}, \mathrm{N}, \mathrm{O}$, and $\mathrm{Fe}$ and also have lines arising from ionization states as high as $\mathrm{N} \mathrm{V}$ (which is found in gas as hot as $3 \times 10^{5} \mathrm{~K}$ ). Unfortunately, ultraviolet observations suffer from the drawback that they must be obtained from space and are therefore limited by the characteristics of the available instrumentation and the stiff competition for its time. At the time of writing, this has essentially meant IUE (the International Ultraviolet Explorer).

cool vs. hot gas. Although data are available on resonance transitions of Al III, Si IV, C IV and N V in the halo, these will not be discussed here. Strictly speaking, these species could well arise in cool gas that is subjected to a strongly ionizing radiation flux. Indeed, whether the highly ionized species observed in the galactic halo arise from photo-ionization or from collisions with thermal electrons is still widely debated. However the nature of the highly ionized species will be discussed elsewhere in this volume.

The discovery of the highly ionized species in the halo focused a great deal of attention on the possible presence of a galactic "corona" with temperatures greater than $10^{6} \mathrm{~K}$ or cooling through $10^{5} \mathrm{~K}$. Relatively little work has been done on what happens to the gas after it cools below $10^{4} \mathrm{~K}$ and how it settles back to the plane. However, even the earliest observations of halo gas by Savage and de Boer (1979) showed that there is at least a factor of ten times more cool gas by mass in the halo than the highly ionized gas, as observed through lines such as C I, O I, Mg I, Mg II, Si II, Al II, Fe II, C II, and so on.

We will discuss the results from observations of the neutral and singly ionized species on the nature of cool gas in the halo, it structure and its kinematics. Ultimately, one hopes that the data will provide insight into the origin and history of halo gas: Is the cool gas former hot gas from a galactic fountain? Or was it removed from the disk to high $z$ ? If so, how? Or is cool halo gas simply a high-z tail to the disk distribution of the interstellar medium?

In the next section we present a brief overview of past and current optical and ultraviolet observational studies of halo gas. Section III highlights some interesting problems, and finally, Section IV discusses the most recent efforts to get more quantitative information from the absorption spectroscopy, particularly in light of new, higher quality instrumentation available. We will not discuss (1) the lines of sight toward the LMC/SMC/SN1987a or (2) Lyman- $\alpha$ absorption studies à la Lockman, Hobbs 
and Shull (1986); these subjects are quite broad and in some cases controversial, and they will be covered elsewhere in this volume.

\section{Resources and Results from Optical and Ultraviolet Studies}

This section does not aim to present the history of optical and ultraviolet absorption studies of halo gas. Many extensive reviews of the history and overall properties of halo gas can be found in the literature, including Savage (1989), de Boer (1989), and references therein. Instead, we merely list some of the most comprehensive sources, and briefly summarize some of the salient results. The list of sources here is by no means exhaustive: it is merely intended to provide a set of references which can be used as a resource for more in-depth work.

\section{TABLE 1 RESOURCES FOR OPTICAL DATA}

Munch and Zirin (1961)

Albert (1983)

Morton and Blades (1986)

Edgar and Savage (1989)

Albert et al. (1991) 1st comprehensive halo survey comp. halo survey (19 זs; $5 \mathrm{~km} / \mathrm{s}$ res.)

halo and X-gal sightlines

complilation from lit : refract. elem. comp. halo survey (66 ts; $5 \mathrm{~km} / \mathrm{s}$ res.)

A. Optical data. Table 1 provides a partial list of references for optical surveys of absorption from halo gas. Next to each reference, comments indicate either the observational techniques, sample selection criteria, or other attributes which make that particular study unique or noteworthy.

To summarize the results of these and other studies, the optical data show that the number of cool clouds continues to increase as the distance above the galactic plane increases, indicating that such clouds can indeed be found in the halo. Interestingly, halo clouds possess velocities that are distinctly different from disk velocities, ranging from about $-50 \mathrm{~km} / \mathrm{s}$ to +20 $\mathrm{km} / \mathrm{s}$. Authors have noted the propensity for negative velocities to be found in halo components.

There is no significant increase in the number of components beyond the lower halo, i.e., there is not any substantial difference in the appearance of absorption spectra against extra-galactic sources compared to halo stars beyond 2-3 kpc above the disk. Halo clouds demonstrate the Routley-Spitzer effect; i.e., the ratio of $\mathrm{Na}$ I to $\mathrm{Ca}$ II decreases in high $\mathrm{z}$-- and larger velocity - clouds. In keeping with this, the refractory elements are seen to have higher scale heights than the non-refractory elements. Interestingly, refractory elements display a smoother distribution: the scatter in the observed correlation 
between distance (or z-distance) and column density is smaller for refractory elements.

Finally, mention should be made of efforts to detect molecules at high latitudes. Absorption from $\mathrm{CH}, \mathrm{CH}+$, and $\mathrm{CN}$ has been detected (DeVries and Van Dishoeck 1988; Welty et al. 1989; Penprase 1991) in stars lying in the direction of high $b$ clouds from the survey of Magnani, Blitz and Mundy (1985). While none of the MBM clouds has been detected at distances beyond about $\mathrm{z}=250 \mathrm{pc}$, they may play an important role in the disk-halo interaction. The absorption data can provide important information on the presence of and the ionization conditions in high latitude molecular clouds.

\section{TABLE 2 RESOURCES FOR ULTRAVIOLET DATA}

Savage and de Boer $(1979,1981)$

Van Steenberg \& Shull $(1988 \mathrm{a}, \mathrm{b})$

Danly (1989)

Kinney et al. (1990)

Danly et al. (1991) 1st UV halo study

disk and halo survey

halo kinematics survey ( 34 irs; $b>40^{\circ}$ )

$X$-gal sightlines

halo survey (57 is; data plotted)

B. Ultraviolet data. In a manner similar to Table 1, Table 2 lists references for ultraviolet absorption studies of halo gas. Some of the best known references on UV absorption data, such as Pettini and West (1983) or Savage and Massa (1987) are not listed because their focus was heavily weighted toward the high ionization species. Again, the samples in some of the surveys included both disk and extra-galactic sources, and again, the list is not exhaustive.

As mentioned earlier, an important result from the ultraviolet studies is that low ionization material is about a factor of ten times more abundant than the high ionization material. Observations at lower resolution toward extragalactic sources also clearly indicate that the low ionization species are more prevalent than the higher ionization species. Interestingly, the Fe II abundance may be enhanced in halo gas.

As was found with the weaker, optical components, halo absorption is commonly found with large negative velocities. However, the velocity extent of the ultraviolet absorption always exceeds the velocity extent of the optical absorption lines, usually by a significant amount: the very lowest column density halo gas (with column densities below the detection limit of the optical lines, but large enough to be detected with the most sensitive UV lines) can commonly be found with total velocity ranges of $\Delta v \sim 130-150$ $\mathrm{km} / \mathrm{s}$ (see Figure 1). The full velocity range of the low ionization UV lines also always exceeds that of the higher ionization lines observed in the same direction, even when comparing lines of similar f-values. 
One very important result from the ultraviolet studies is that the gas toward the North Galactic Pole (NGP) displays very different characteristics than the gas toward the South Galactic Pole (SGP). This asymmetry may have important implications for all models for halo gas production and maintenance. It is discussed in more detail in the next section. Finally, the data show considerable differences along different lines of sight suggesting structure on all scales. Comparison with $21-\mathrm{cm}$ observations of clouds at high latitudes shows very good correlation and suggest that the interpretation of data along individual lines of sight may be best understood through a multifrequency approach.

\section{Some Interesting Problems}

A. Infalling Gas Toward the NGP. The preference for negative over positive velocities toward the NGP is clearly observed in both the optical and ultraviolet data (see Figure 1). Toward the the NGP, absorption from infalling gas with velocities more extreme than $v=-70 \mathrm{~km} / \mathrm{s}$ is found toward nearly all stars with $\mathrm{z}>2 \mathrm{kpc}$, while toward the SGP, none of the stars show absorption beyond $-70 \mathrm{~km} / \mathrm{s}$. In fact, toward the SGP, there is no evidence at all for any preference toward infalling gas (i.e. with negative velocities) over outflowing gas (i.e. with positive velocities). Based on the SGP data alone, one would be hard pressed to find evidence for the return of cool gas to the plane in a galactic fountain flow.

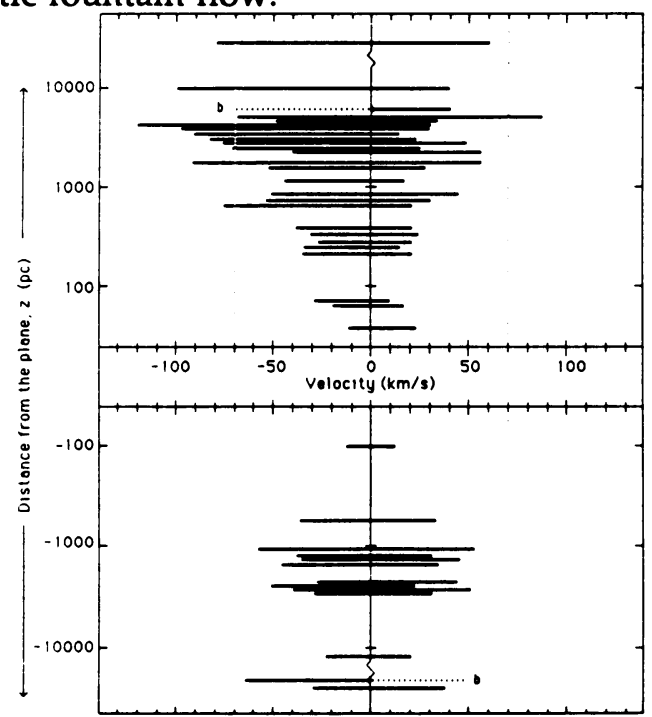

Figure 1. A plot of the absorption widths of the most sensitive low ionization lines as a function of position of the background object. The velocities are measured from composites of the Si II $\lambda 1260, \mathrm{C}$ II $\lambda 1334$, and the $\mathrm{OI} \lambda 1302$ lines. Absorption beyond $z<-70 \mathrm{~km} / \mathrm{s}$ is only seen toward the NGP, primarily in stars beyond $z=2.4 \mathrm{kpc}$. The only stars with $\mathrm{z}<2.4 \mathrm{kpc}$ that show absorption in this range lie behind the IV arch (see SIIIc). Lines of sight toward the SGP show no preference for infall of cool gas from the halo. 
Most of the infalling gas in the north is concentrated in structures located primarily in the second quadrant of galactic longitude (i.e. $1=90^{\circ}$ to $\left.180^{\circ}\right)$. Some of the clouds possess very large velocities $(\mathrm{v}<-100 \mathrm{~km} / \mathrm{s})$ and make up the well known HVC Complexes C I, C III, and A (see Figure 2a; see also Wakker 1989 and his review in this volume). Also in this direction lie the IVC Complexes C II and M II which have velocities $-90 \mathrm{~km} / \mathrm{s}<\mathrm{v}<-50$ $\mathrm{km} / \mathrm{s}$ (Figure $2 \mathrm{~b}$ ). Interestingly, the velocities of the infalling clouds appear to correlate roughly with distance in the sense that the most distant gas has the most extreme velocities. This is just the opposite of what would be expected if the gas were accelerating on ballistic orbits in the galactic potential. The properties of the infalling cloud complexes present unique problems in themselves and are discussed separately in the following two sections.

If any progress is to be made on the theory of galactic halo gas, one must first understand the nature of the infalling gas toward the NGP: its presence dominates our view of halo gas kinematics. The confirmation and refinement of a galactic fountain model strongly depends on whether this feature is a product of such a flow, or whether it can be understood in terms of an entirely separate, independent mechanism.

B. Where are the Elusive High Velocity Clouds? A complete and upto-date review of all attempts to detect any HVCs in absorption can be found in Van Woerden, Schwarz and Wakker (1989) and will not be repeated here. In summary, while HVCs have been detected in absorption toward extragalactic sources, no positive, confirmed detection of an HVC has yet been made in absorption toward a galactic source which can provide an upper limit on its distance. In this instance, "high velocity" refers to $|\mathrm{v}|>100 \mathrm{~km} / \mathrm{s}$.

Songaila, Cowie and Weaver (1988; hereafter SCW) have claimed absorption detections of both Complex A and Complex C. They place an upper limit on the z-distances to the complexes at $1.1 \mathrm{kpc}$ (Complex A) and 1.7 kpc (Complex C). Their claim has been disputed for several years. New evidence casting more doubt on their result has surfaced since the last review (Van Woerden, Schwarz and Wakker 1989) and will therefore be summarized here.

Lilienthal, Meyerdierks and de Boer (1990) show that the feature claimed by SCW as high velocity absorption toward Complex $\mathrm{A}$ is probably a misidentified stellar Ti II line blended with the stellar $\mathrm{Ca}$ II $\mathrm{K}$ line (see contribution by de Boer, this volume).

SCW claim absorption from Complex $C$ can be seen in the spectrum of the RR Lyrae star BT Dra. However, the saturated stellar lines in RR Lyrae photospheres (including Ca II lines) have long been known to be highly complicated, demonstrating bumps, wiggles and splitting depending on the phase of the variable (Preston and Paczynski 1964). For example, the saturated $\mathrm{Ca} \mathrm{K}$ spectrum shown in SCW is about 10 times broader than the $\mathrm{Na} \mathrm{D}$ spectrum toward the same star published earlier (Songaila et al. 1985), and and the dips and troughs seen over the entire stellar line are the result of 
complicated optical depth effects as a function of the variable's phase, averaged over the length of the exposure. Since SCW give no information about exposures or phase information about the variables it is impossible to interpret their observations. No claims as to the distance to the HVCs based on these data can be substantiated unless a more careful analysis of the stellar spectrum as a function of phase and/or using other, less ambiguous species is carried out.

Evidence that in fact Complex $C$ lies beyond $1.7 \mathrm{kpc}$ comes from the non-detections observed by Danly (1989; data can be found in Danly et al. 1991) in the UV spectra of two stars which also lie toward Complex C at about that same distance. The UV lines are about two orders of magnitude more sensitive than the optical lines and should show strong absorption if the Complex indeed lies in front of the stars.

One way in which a non-detection might occur even if the Complex lies in front of the stars is if the gas distribution is patchy on scales smaller than the smallest radio telescope beam used to observe the $21-\mathrm{cm}$ emission from the HVC. If such small scale structure exists, it is possible that the lineof-sight to the background source passes through a local "hole" in the gas distribution. Preliminary results from new observations using the Westerbork Radio Synthesis Telescope of the gas toward stars from Danly (1989) lacking HVC detections show that patchiness is not likely to be able to account for the non-detection (Danly, Wakker and Schwarz 1991).

Another possible explanation for the non-detections is that through some combination of abundance, depletion and ionization, the number of absorbing ions relative to $\mathrm{N}_{\mathrm{H}}$ is too low to be detected in absorption. One way to test this alternative is by observing an extra-galactic source which lies near a galactic source showing a non-detection. An interesting example of this is seen in the case of the non-detection in the $z=2.9 \mathrm{kpc}$ halo star BD +56 1411 (Danly and Kuntz, 1991) and the detection toward QSO MK 106 (Schwarz, Wakker and Van Woerden 1991) which lies less than $5^{\circ}$ from BD +56 1411 in the direction of Complex A. Significant variations in abundance over relatively small scales within a cloud would be required to reconcile these observations if the HVC resided in front of the star. While this cannot be ruled out, abundance variations over such scales would be remarkable, considering the mixing that is likely to occur in the clouds. Complex $\mathrm{A}$ is probably beyond $z=2.9 \mathrm{kpc}$, although the question of patchiness still needs to be investigated along this line of sight.

A more general statement of all of the above arguments can be made on statistical grounds: the growing numbers of detections of HVCs in QSOs and the growing numbers of non-detections in galactic sources lying several kpc above the disk make it highly improbable that the non-detections toward halo stars are merely chance super-positions along lines of sight with unfavorable conditions.

The uncertainty in stellar distance determinations remains a serious limitation of absorption studies. Any firm, quantitative determinations will be limited by the quality of the stellar analysis. But at the present time, we 


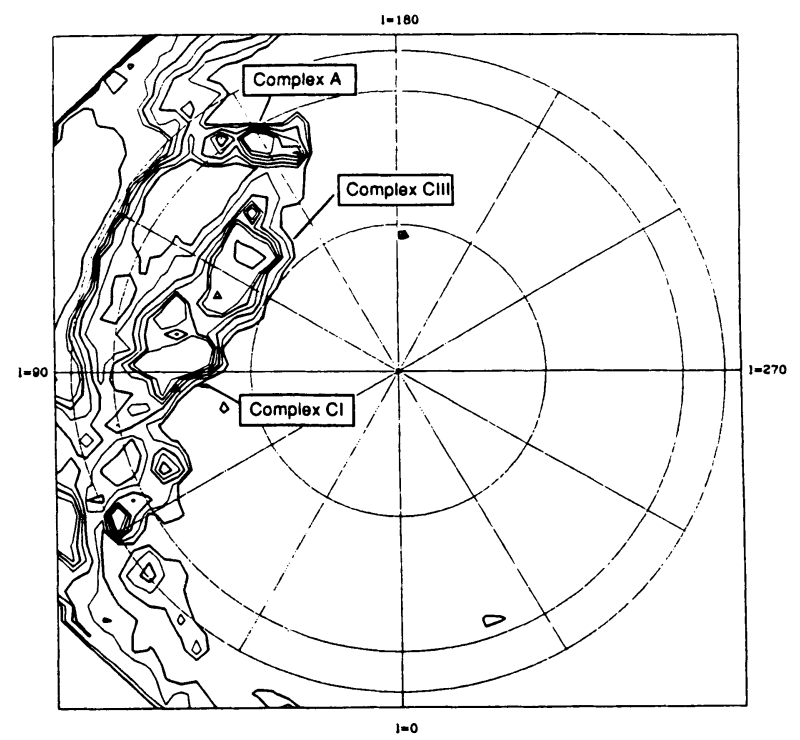

Figure 2a. Contour plot of 21-cm emission seen toward the NGP over the velocity ranges of -130 $\mathrm{km} / \mathrm{s}$ to $-160 \mathrm{~km} / \mathrm{s}$ (HVCs). Contour levels are $\mathrm{a}, 2,4,6,8,10,20,40$, and $60 \times 10^{18} \mathrm{~cm}^{-2}$. The HVC Complexes are clearly marked, as are the stars from Danly (1989) which provide lower limits from non-detections and BT Dra, the star claimed by SCW to show HVC absorption.

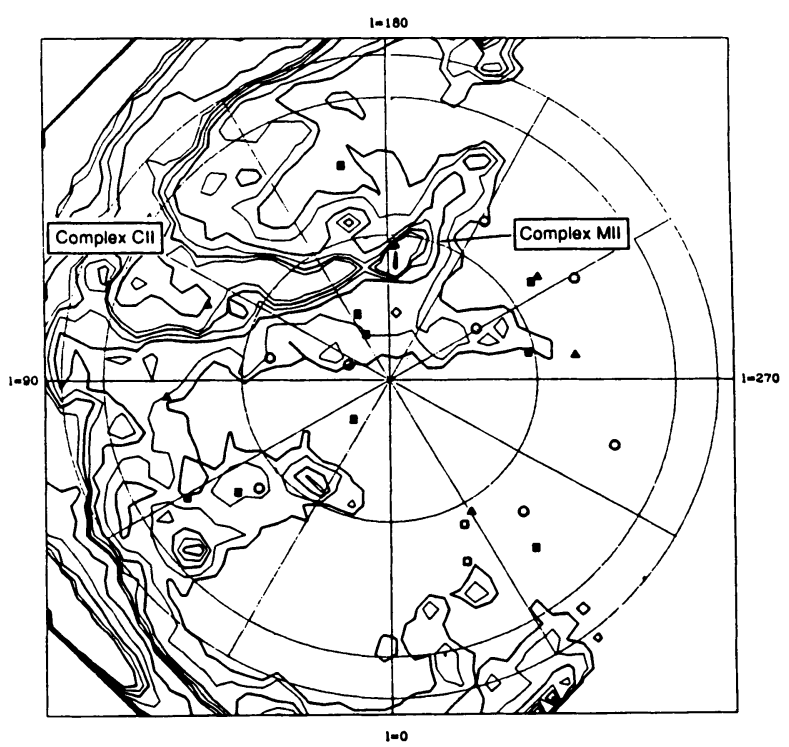

Figure $2 \mathrm{~b}$. Same as Figure 2a except it shows the velocity range of $-70 \mathrm{~km} / \mathrm{s}$ to $-85 \mathrm{~km} / \mathrm{s}$. The symbols show the positions of stars from the survey by Danly (1989). The solid symbols show absorption over this range of velocities and the open symbols do not. The symbol shapes represent distance: circles have $z<700 \mathrm{pc}$, triangles have $700 \mathrm{pc}<z<2400 \mathrm{pc}$, and squares have z>2400 pc. 
conclude that no absorption detection of an HVC has yet unambiguously been made which provides a distance estimate for HVCs -- their distances are still unknown. We further conclude that, in fact, there is growing evidence to support the view that the NGP HVC complexes are beyond the lower halo: i.e. beyond about $2 \mathrm{kpc}$. Any advancement of our understanding of the origin of the infalling HVC complexes and their relation to overall halo gas models will rest on determining their distances; most further analyses of their physical properties rely on a knowledge of this important and undetermined parameter.

C. Infalling Gas with Intermediate Velocities. The first evidence that the northern galactic sky was covered with infalling IVCs was from the $21-\mathrm{cm}$ survey of Wesselius and Fejes (1973). Amazingly, in the 17 years since its publication, Wesselius and Fejes' paper has only been cited 25 times, and of those, only a handful advance any new results on the gas. The IVCs have been terribly overlooked.

One fascinating property of the northern IVCs is the strong positional anti-correlation with the low-velocity disk gas: the IVCs are most concentrated in directions where emission from local disk gas is weakest. This anti-correlation is suggestive of gas removal or displacement, but the details have never been fully worked out nor have the implications been clearly understood.

Individual IVCs or IVC complexes are not as well known or well studied as the HVCs. However, most of the NGP IVCs are contained in Complexes C II and M II which have velocities between roughly $-50 \mathrm{~km} / \mathrm{s}$ and $-90 \mathrm{~km} / \mathrm{s}$-- their names were originally coined when they were studied as part of the HVC complexes. We refer to these Complexes and their associated emission as the "IVC arch" (see Figure 2b). The distance to the arch is somewhere between $z=300 \mathrm{pc}$ and $\mathrm{z}=1700 \mathrm{pc}$, although $\mathrm{M}$ II may be as close as 700 pc (Danly 1989 for complete discussion). Fortunately, its proximity suggests that, with further study, the distance to the arch will be even better constrained.

The IVC arch has many intriguing characteristics. It contains most of the mass in the local halo, of order $10^{5} \mathrm{M}_{\mathrm{o}}$ (Danly 1989). It contains more than 1051 ergs of kinetic energy; some unusual and highly energetic event must have occurred if it was kicked out from the disk. Indeed, its morphological structure suggests it may have been related to the disk. On the other hand, perhaps the IVCs are former HVCs which have been decelerated as they fall back to the disk. As such the study of their physical characteristics, such as abundances and ionization, may provide important insights into the HVC phenomenon. Again, the spatial coincidence of the HVCs and the IVCs suggests there may indeed be a link. In any case, the unique situation of the IVC arch between local disk gas and the HVCs, in both space and velocity, makes it particularly fascinating.

An in-depth study of the physical properties of the IVC arch has only recently begun (Danly and Kuntz 1991), but early results suggest that the 
abundances are near solar. Further, the ionization structure is different in different directions along the complex. Interestingly, local densities along the arch are high enough to permit the excitation of the fine structure lines of $C$ II. Further study, including investigations using other observational techniques, is needed for a more complete understanding of this feature.

Equally intriguing is the IV gas not in the IV arch. Absorption near -70 $\mathrm{km} / \mathrm{s}$ can be seen in nearly all stars (and in all four quadrants of galactic longitude) beyond $\mathrm{z}=2.4 \mathrm{kpc}$, but lying well off the $\mathrm{N}_{\mathrm{H}}=10^{18} \mathrm{~cm}^{-2}$ contour. This is probably because the UV lines are sensitive to column densities of gas that are too low to be detected in $\mathrm{H} \mathrm{I}$. Absorption at $-70 \mathrm{~km} / \mathrm{s}$ toward stars with $\mathrm{z}<2.4 \mathrm{kpc}$ can only be seen in stars behind the arch.

Equally remarkable is the $21-\mathrm{cm}$ emission near $-50 \mathrm{~km} / \mathrm{s}$ that covers the entire sky at a level of $\mathrm{N}_{\mathrm{H}}=10^{18} \mathrm{~cm}^{-2}$ ! Absorption measurements show that the distance to the $-50 \mathrm{~km} / \mathrm{s}$ gas is well determined to between 370 and $800 \mathrm{pc}$ above the disk. The $-50 \mathrm{~km} / \mathrm{s}$ gas alone contains an additional $10^{5} \mathrm{M}_{\mathrm{o}}$ and of order $10^{51}$ ergs of kinetic energy.

In addition to being intrinsically interesting, the IVCs between about 0.4 and $2 \mathrm{kpc}$ above the disk can serve as "passive" probes of the environment in the lower halo and the disk-halo interaction. For example, at these distances, the IVCs lie well above the protective shielding of the disk gas and are subjected to both the escaped galactic and the inter-galactic radiation fields (see next section). The IVCs may play an important role in searching for evidence for the existence of chimneys or other galactic fountain-related phenomena. Additionally, the IVCs may be perfectly situated to interact with streaming galactic cosmic rays and might be observable through gamma-ray production.

Finally, the IVCs may be used in the study of the dynamics of the gas deceleration. Probably in the case where gas slows from HVC velocities to -70 $\mathrm{km} / \mathrm{s}$, and certainly in the case of the slowing from $-70 \mathrm{~km} / \mathrm{s}$ to $-50 \mathrm{~km} / \mathrm{s}$ to the near $-0 \mathrm{~km} / \mathrm{s}$ velocities of the disk, there is not enough material to provide sufficient viscous drag to slow the gas. Some other dynamical processes (such as magnetic fields) may be at work. The impact of the falling clouds as they arrive at the disk will be very different depending on whether they shock the disk with their full $10^{51}$ ergs of kinetic energy (Tenorio-Tagle 1981; TenorioTagle et al. 1988) or whether they slowly settle on to the disk.

D. The ionization problem. Most photo-ionization models for galactic halo gas require an ionization rate of about $\Gamma \sim 10^{-11} \mathrm{sec}^{-1}$ in order to produce the observed C IV and Si IV. An ionization rate on this order is supported by observations of the vertical column of $\mathrm{H}^{+}$integrated along the entire pathlength toward the NGP (Reynolds 1989) and by the C IV diffuse emission observed by Martin and Bowyer (1989) toward the NGP (G. Field, private communication). Looking outside the galaxy, the sharp edges of galaxies observed by Van Gorkom et al. (1991; see also Sancisi 1987) at limits of $\mathrm{N}_{\mathrm{H}}$ of 
about $2 \times 10^{19} \mathrm{~cm}^{-2}$ can also be accounted for by an ionization field on this order.

Recent observations of $\mathrm{H}-\alpha$ fluxes from the HVCs lying $>2 \mathrm{kpc}$ above the disk (see above) have shown that the ionization rate of hydrogen in the clouds is roughly two orders of magnitude lower -- about $\Gamma \sim 10^{-13} \mathrm{sec}^{-1}$ (Songaila, Bryant and Cowie 1989, Kutyrev and Reynolds 1989). A lower ionization rate would also be required to be consistent with the observations by Danly (1989) of O I observed toward stars beyond $2.4 \mathrm{kpc}$. Since the ionization potential of Oxygen is very similar to Hydrogen $(\Phi=13.6 \mathrm{eV})$ the two species track each other very well. The observation of $\mathrm{O} I$ in the halo in directions where $21-\mathrm{cm}$ observations are not sensitive enough to detect the neutral gas suggest that neutral clouds with column densities between about $8 \times 10^{16} \mathrm{~cm}^{-2}$ and $10^{18} \mathrm{~cm}^{-2}$ reside well above the shielding galactic disk. The presence of neutral clouds at these low column densities is inconsistent with the view that an extragalactic radiation field strong enough to account for the sharp edges observed on external galaxies reaches the lower halo $(\sim 2 \mathrm{kpc})$.

The controversy raised by these observations reminds us that very little is understood about the nature of the cool and neutral gas in the galactic halo: how can the observed amounts of the cool gas form and survive in an environment subjected to either (or perhaps even both) ionization from the extra-galactic radiation field and/or coronal gas from a fountain? Any complete theory of galactic halo gas production must account for all the observed phases.

\section{Future Work: The Quest for More Quantitative Results}

The greatest limitations on absorption studies are imposed by curve-ofgrowth (c.o.g.) effects coupled with low instrumental resolution. The problems are not as severe for the Li-like ions (such as the high ionization species of C IV, Si IV, N V, O VI) which have doublet transitions of $2 s^{2} \mathrm{~S}$ $2 \mathrm{p}^{2} \mathrm{P}^{0}$ or $3 \mathrm{~s}^{2} \mathrm{~S}-3 \mathrm{p}^{2} \mathrm{P}^{0}$ for which fairly good quantitative estimates of column densities can be achieved using the doublet ratio method. But both line saturation and the uncertainties in f-values for species commonly found in neutral or weakly ionized gas make it difficult for quantitative measurements of interstellar column densities to be achieved with much accuracy.

Some authors have attempted to derive quantitative information from IUE data despite its comparatively low resolution $\left(R=10^{4}\right)$. Van Steenberg and Shull $(1988 a, b)$ derive column densities using a single-component c.o.g. analysis, relying on the result by Jenkins (1986) that such an approximation is valid provided the absorbing ensemble of lines has a distribution of strengths that is not highly irregular (e.g. bi-modal). The derived column densities have large associated uncertainties. Additionally, a single-component c.o.g. analysis provides no information on component-to-component variations that are almost certain to be present along lines of sight which pass through regions possessing different physical conditions. 
Danly and Blades (1989) have selected pairs and groups of stars for which very high quality, high resolution optical $\mathrm{Ca}$ II data are available from the survey of Albert et al. (1991). The selected lines of sight show clear multicomponent structure. Using the optical data as a guide to model the cloud velocity structure, they are able to determine column densities for the low ionization species with an accuracy of a factor of two, in all but the very central, near $-0 \mathrm{~km} / \mathrm{s}$ portion of the profile. They find clear variations in ion ratios from one component to the next along a single line of sight.

Using a different technique, Savage, Massa and Sembach (1990) have carried out an in-depth analysis of the line of sight toward HD 163522 which lies $9 \mathrm{kpc}$ away in the direction $1=350^{\circ}$ and $b=-9^{\circ}$. In this direction, galactic rotation spreads out the absorption profile over a range of about $160 \mathrm{~km} / \mathrm{s}$. They find that under these conditions, saturation in the highly ionized species is minimized and direct estimations of column densities are obtainable by employing what is effectively a doublet ratio method carried out as a function of velocity. They find that applying the same method to many Fe II lines (where the f-values are less accurately determined) provides useful numbers in the line wings, though saturation in the core is still a problem.

Minimizing the limitations imposed by c.o.g. effects requires several lines of the same species, spread over a wide range in f-values. Observing in the UV part of the spectrum helps in this regard because of the large numbers of lines available. Even greater opportunities will be made available with the launch of Lyman-FUSE which will probe the far-UV portion of the spectrum at resolutions of $3 \times 10^{4}$ (although Lyman-FUSE will be best suited for the study of more highly ionized species). Finally, all quantitative measures will only be as accurate as the atomic data (i.e. f-values) available.

Overcoming the difficulties introduced by poor instrumental resolution will be made better by the next generation of space instruments: GHRS on HST will be able to perform spectroscopy on halo stars at a resolution of $10^{5}$. However, GHRS is only able to record one order of the echelle spectrum at a time, requiring a separate observation for nearly every species desired. For example, a 10th magnitude BO star (which with IUE takes about 2 hours to obtain an $R=1.2 \times 10^{4}$ spectrum with $S / N \sim 10: 1$ ) will require about 3.5 hours of exposure time to get 10 selected species with a $S / N$ of about 30:1. The situation will improve dramatically, however with the second generation Space Telescope Imaging Spectrograph (STIS) which will be able to observe with $R \sim 140,000$ and which will have a large imaging detector capable of capturing most of the important interstellar species with one exposure. The same 10th magnitude B0 star will require a 1-hour exposure to produce a spectrum complete over the range $\lambda \lambda 1050-1700$ with a $\mathrm{S} / \mathrm{N}$ of about 100:1.

As observational techniques improve, progress in the study of halo gas will be made along two fronts: (1) the more quantitative analysis of interstellar data toward known, bright halo stars, and (2) an expansion of the roster of background sources to much fainter magnitudes. The number of 
bright, blue halo sources is limited, and many of the most suitable objects have been observed with IUE. Future progress can be made by re-analyzing those lines of sight at higher resolution to provide significantly more accurate column densities on a component by component basis. Importantly, advancements in the theory of halo gas are also needed in order to derive physical characteristics of the gas from the diagnostic line strengths and column density ratios.

Progress can also be made by significantly increasing the sampling of halo sightlines by identifying additional background sources. They can be either galactic or extra-galactic, although galactic sources have the added benefit of providing distance information for halo clouds. Surveys reveal a significant population of faint, blue halo stars whose characteristics are not well-understood and whose distances are therefore unknown. Improvements in their photospheric analyses could lead to a substantial increase in the number of halo stars identified which can serve as probes. The ability to observe to fainter magnitudes also means that blue horizontalbranch stars in globular clusters can be employed. Finally, extragalactic sources, such as QSOs, could provide a considerable increase in the sightlines available to sample halo gas. For example, STIS will be capable of obtaining a spectrum toward a $B=16^{\mathrm{m}}$ QSO with $\mathrm{S} / \mathrm{N} \sim 15: 1$ in its $\mathrm{R}=2 \times 10^{4}$ mode in about an hour. At a surface density on the sky of about $0.02 \mathrm{QSO}$ per square degree for $B<16^{m}$ (Schmidt and Green, 1983), the use of QSOs could dramatically increase the available data on halo gas over the longer term.

Future developments in absorption studies of halo gas rely on very sensitive instruments outside the Earth's atmosphere. A serious trade-off is made between instrumental capabilities and long exposure times: it is unlikely that large, multi-purpose space telescopes would be used to survey the galactic halo the way IUE was employed in the past. Therefore, planning observations to make most efficient use of the spacecraft time is essential: the most effective programs will formulate predictions very carefully, identifying the most critical lines to be observed which will discriminate among competing theories.

ACKNOWLEDGEMENTS The author thanks Elise Albert, Keith Ashman and Colin Norman for careful readings of the manuscript. Richard Green also provided valuable information regarding the performance of STIS.

\section{REFERENCES}

Albert, C. E., 1983, Ap.J., 272, 509

Albert, C.E., Blades, J.C., Morton, D.C., and Proulx, M., 1991, in preparation Danly, L.,1989, Ap.J., 342, 785

Danly, L., and Blades, J.C., 1989, in Proc. from the IAU Colloq. \#120 The Structure and Dynamics of the Interstellar Medium, G. Tenorio-Tagle, M. Moles, and J. Melnick eds., p. 408 
Danly, L. and Kuntz K.D., 1991, in preparation

Danly, L., Savage, B.D., Lockman, F.J., and Meade, M.R. Ap.J. Suppl., in press Danly, L., Wakker, B., and Schwarz, U., 1991, in preparation

de Boer, K.S., 1989, IAU Colloq. \#120 op. cit., p. 432

de Vries, C.P. and Van Dishoeck, E.F., 1988, Astron. Ap., 203, L23

Edgar, R.J. and Savage, B.D., 1989, Ap.J., 340, 762

Jenkins, E.B., 1986, Ap.J., 304, 739

Kutyrev, A.S. and Reynolds, R.J., 1989, Ap.J., 344, L9

Kinney, A., Bohlin, R., Blades, J.C., and York, D.G., 1990, Ap.J., in press

Lilienthal, D., Meyerdierks, H., and de Boer, K.S., 1990, Astr. Ap, in press

Lockman, F.J., Hobbs, L.M., and Shull, M., 1986, Ap.J., 301, 380

Magnani, L., Blitz, L., and Mundy, L, 1985, Ap.J., 339, 244

Martin, C. and Bowyer S., 1989, Ap.J., 350, 242

Morton, D.C. and Blades, J.C., 1986, M.N.R.A.S., 220, 927

Munch, G., and Zirin, H. 1961, Ap. J., 133, 11

Penprase, B.E., 1991, Ph.D. Thesis, University of Chicago

Pettini, M. and West, K.A., 1982, Ap.J., 260, 561

Preston, G.W. and Paczynski, B., 1964, Ap.J., 140, 181

Reynolds, R.J., 1989, in Proc. IAU Symp. \#139, in press

Sancisi, R.,1987, in STScI Symposium Series \#2 on QSO Absorption Lines

Savage, B.D., 1989, in Proc. ASP Centennial, Berkeley, in press

Savage, B.D. and deBoer, K.S., 1979, Ap.J., 230, L77

Savage, B.D. and deBoer, K.S., 1981, Ap.J., 243, 460

Savage, B.D. and Massa, D.M., 1987, Ap.J. 314, 380

Savage, B.D. Massa, D.M., and Sembach, K., 1990, Ap.J., 355, 114

Schmidt, M., and Green, R., 1983, Ap.J., 269, 352

Schwarz U., Wakker B. and Van Woerden H.,1991, in preparation

Songaila, A., Bryant, W., and Cowie, L.L., 1989, Ap.J., 345, L71

Songaila, A., Cowie, L. L., and Weaver H. 1988, A n. J., 329, 580

Songaila, A., York, D. G., Cowie, L. L., and Blades, J. C.,1985, Ap. J., 293, L15

Tenorio-Tagle, G. 1981, Astr. Ap., 94, 338

Tenorio-Tagle, G., Franco, J., Bodenheimer, P., and Rozyczka, M., 1988, Astr. Ap., 179, 219

Van Gorkom J., Van Albada, T.S., Cornwell, T., and Sancisi, R., 1991, in prep.

Van Woerden H., Schwarz U., and Wakker B., 1989, IAU Colloq. \#120 op. cit. p. 389

Wakker, B., 1989, Ph.D. Thesis, University of Groningen

Van Steenberg, M.E., and Shull, J.M., 1988a, Ap.J. Suppl., 67, 225

Van Steenberg, M.E., and Shull, J.M., 1988b, Ap.J., 330, 942

Welty, D.E., Hobbs, L.M., Blitz, L., and Penprase, B.E., 1989, Ap.J., 346, 232

Wesselius, P.R., and Fejes, I. 1973, Astr. Ap., 24, 15 